\title{
Perioperative Nursing of a Case of Right Temporal Lobe Brain Abscess Ruptured into the Ventricle
}

\author{
Xiaoqun Chen, Shuo Yang, Qinqin Zhao, Zhihuan Zhou* \\ Department of Neurosurgery, Sun Yat-sen University Cancer Center, State Key Laboratory of Oncology in South China, \\ Collaborative Innovation Center for Cancer Medicine, Guangzhou, China \\ Email: chenxiaoq@sysucc.org.cn, *zhouzhh@sysucc.org.cn
}

How to cite this paper: Chen, X.Q., Yang, S., Zhao, Q.Q. and Zhou, Z.H. (2021) Perioperative Nursing of a Case of Right Temporal Lobe Brain Abscess Ruptured into the Ventricle. Journal of Cancer Therapy, 12, 233-239.

https://doi.org/10.4236/jct.2021.125022

Received: April 4, 2021

Accepted: May 10, 2021

Published: May 13, 2021

Copyright (อ 2021 by author(s) and Scientific Research Publishing Inc. This work is licensed under the Creative Commons Attribution International License (CC BY 4.0).

http://creativecommons.org/licenses/by/4.0/

\begin{abstract}
Objective: To explore the perioperative nursing method of brain abscess breaking into the ventricle. Methods: By reviewing the clinical data of 1 case of right temporal lobe brain abscess into the ventricle, the effective nursing methods were summarized. Results: The patient's condition was advanced quickly, and the diagnosis was treated in time to avoid brain hernia, but the treatment was difficult because of the critical condition. Conclusion: Brain abscess is a very serious intracranial infectious disease. It is of great significance to observe the changes in patients' condition and take effective nursing measures.
\end{abstract}

\section{Keywords}

Brain Abscess, Ventricle, Perioperative Nursing

\section{Introduction}

Brain abscess is an infectious disease of the central nervous system caused by suppurative bacteria, and its main clinical symptoms include fever, headache, and focal neurological impairment [1]. Brain abscess can directly destroy brain tissue, and its pathological process includes the stage of encephalitis, the stage of suppuration, and the stage of capsule formation [2]. After the formation of the abscess capsule, should be timely surgical treatment, otherwise, after the abscess rupture, it will endanger the life of the patient. Rupture of brain abscess into the ventricle is a serious complication of brain abscess, with an incidence of $10 \%$ $31 \%$ and a mortality rate of nearly $80 \%$ [3]. The clinical manifestations of brain abscess rupture are not obvious and the disease progresses quickly, leading to 
difficulties in treatment. In January 2020, a patient with a right temporal lobe cerebral abscess ruptured into the ventricle was admitted to our department. Now the treatment and nursing process is reported as follows.

\section{Information and Methodology}

\subsection{Clinical Information}

General information: The patient, a 76-year-old female, presented with headache 4 days before admission, mainly in the right frontotemporal region. The patient had no symptoms of nausea, vomiting, convulsions, and dyskinesia of limb movement. CT scan of the head showed mixed iso-hypodense shadows in the right temporal lobe, a quasi-circular shape with a diameter of about $2 \mathrm{~cm}$, and peripheral edema. "Right temporal lobe tumor" was planned to be admitted to our department in the outpatient department. Physical examination: T: $37.8^{\circ}$, BP: 124/84mmHg, P: 82 times/min, R: 19 times/min, KPS: 70. The patient was conscious, in an autonomous position, with normal development, medium nutrition, cooperative physical examination, and pertinent response. The patient's bilateral pupils are equally large and equally round, sensitive to light reflection, with a diameter of about $3 \mathrm{~mm}$. Physiological reflex is present, but pathological reflex is not elicited. The muscle strength of the limbs is grade $\mathrm{V}$, and the muscle tension is not high. The superficial sensation of the whole body was not obvious, the meningeal irritation sign was negative, pathological sign (-), cerebellum (-).

\subsection{Treatment}

After admission, all examinations were completed. The patient's headache and body temperature increased, and she was given mannitol dehydration treatment, anti-infection (Rocephin $2 \mathrm{~g} / \mathrm{d}$ ), and other symptomatic treatment. On the 4th day after admission, the patient showed drowsiness, uncooperative physical examination, positive meningeal stimulation sign, elevated blood image, elevated blood pressure, and hyponatremia. She continued to receive anti-infection treatment and was given to lower blood pressure and correct hyponatremia at the same time. Re-examination of head CT indicated that there were multiple low-density clumps in the right temporal lobe with large surrounding edema. Considering the possibility of brain abscess, anti-infection treatment should be strengthened (MeropenemforInjection $1 \mathrm{~g} / \mathrm{d}$ ) and the condition should be closely observed. On the 6th day after admission, the patient's GCS was 9, and respiratory tract management was strengthened and bedside sputum aspiration was performed. On the 8th day after admission, cerebral abscess puncture and drainage combined with tracheotomy were performed under general anesthesia, and the abscess cavity drainage tube was indwelling during the operation. After the operation, anti-infection, drainage, gastric care, nutrition, and other treatments were continued. The result of brain pus culture showed that: Gram-positive cocci. According to the drug sensitivity results, antibiotics (vancomycin $1 \mathrm{~g} / \mathrm{d}$ ) were replaced. During the treatment, multiple cerebrospinal fluid tests and imaging 
reexaminations were carried out, and the treatment plan of antibiotics was adjusted. The abscess cavity was removed after external drainage for 1 week, and the lumbar cistern drainage tube was indwelling. Two weeks after the operation, the brain $\mathrm{CT}$ and chest CT results showed that: the right temporal lobe was a low-density mass with large surrounding edema, which was consistent with a brain abscess, with no significant change in scope compared with before. The family members requested to be transferred back to the local hospital for further treatment and discharged.

\section{Nursing Methods}

\subsection{Preoperative Care}

\subsubsection{Observation}

Closely observe the changes of the disease and report to the doctor in charge in time. The course of brain abscesses is fast and complicated. Headache is one of the common clinical manifestations in patients with brain abscess [4]. When the patient has an exacerbation of headache, rapid breathing, elevated blood pressure, or changes in consciousness, indicating that the condition is aggravated, the brain abscess may rupture, should inform the doctor in time [5], timely treatment.

\subsubsection{Dietary Care}

According to the patient's condition and nutritional risk screening and evaluation, give dietary guidance, eat high protein, high calorie, digestible food, if necessary, according to the doctor's instructions to intravenous high nutrition treatment, improve the nutritional status of patients.

\subsubsection{Control of Infection}

Closely observe the patient's body temperature change, body temperature rise, give physical cooling or drug cooling, and record the cooling effect, according to the doctor's instructions to use antimicrobial drugs, timely record the efficacy of drugs.

\subsubsection{Psychological Nursing}

Patients have severe headache and continuous fever, the rapid progress of the disease, prone to pain and anxiety, nurses should pay attention to the psychological status of patients, give psychological guidance and support, so that patients and their families to build up the confidence to overcome the disease, Actively cooperate with treatment.

\subsection{Postoperative Care}

\subsubsection{Observation}

Continuous ECG monitoring and low flow oxygen inhalation to strengthen ward inspection. Intracranial hemorrhage is easy to occur $24-48$ hours after craniotomy. 3 - 7 days after the operation is the peak period of cerebral edema. It is necessary to closely monitor the vital signs of patients, dynamically judge the 
GCS score of patients, grasp the changes of the disease in time, and prevent the occurrence of brain hernia.

\subsubsection{Care of Tracheotomy}

The nursing tracheotomy of tracheotomy is an invasive and invasive operation, which is prone to complications such as extubation, subcutaneous hematoma, bleeding, or incision infection. The room temperature should be maintained at $20^{\circ} \mathrm{C}-22^{\circ} \mathrm{C}$, humidity $40-50$ per cent [6]. Use sterile bandages to fix the tracheal cannula, maintain the appropriate tightness according to the patient's condition, keep the tracheal cannula in the positive position, avoid the displacement of the end of the tracheal cannula, and press the inner wall of the trachea to cause loss and bleeding. The endotracheal tube should be cleaned and disinfected regularly, and the sputum should be replaced in time. Airbag pressure is monitored in each class. The Chinese Medical Association recommends that the airbag pressure should be kept at $25-30 \mathrm{cmH}_{2} \mathrm{O}$, to give full play to the role of the airbag, close the airway, and fix the catheter relatively [7]. To avoid gas leakage caused by the displacement of the trachea cannula or excessive pressure on the inner wall of the trachea bleeding. Trachea incision needs to use aseptic dressing change bag, according to the patient's sputum and incision conditions to change medicine, 3 times a day, during the process of dressing change strictly abide by the principle of aseptic operation, gentle action, avoid pulling the patient's incision skin, Cause incision bleeding. According to temperature and humidity in the ward and the viscosity and quantity of the patient's sputum, the humidification nursing of the trachea was done well, and the artificial nose was replaced in time every day to ensure the effect of airway humidification.

\subsubsection{Pipeline Care}

After the operation, patients should be treated with a tracheotomy, indwelling central venous catheter, pus drainage tube, lumbar cistern drainage tube, catheter, and stomach tube. Fix the drainage tube properly and keep the drainage tube unobstructed. Observe and record the color, character, and quantity of the drainage fluid in each class, regularly keep the drainage fluid for examination, and use antibiotics reasonably according to the results of cerebrospinal fluid bacterial culture and drug sensitivity. Observed the central venous catheter dressing and tracheotomy dressing loose, bleeding or exudate, timely change dressing. When nursing the drainage tube and changing the wound dressing, strictly follow the principle of aseptic operation.

\subsubsection{Skin Carer}

The nurse should patiently explain to the family the importance of preventing pressure sores. After the operation, the nurse should strengthen the rollover and pat the back, turn over and pat the back every 2 hours, and use the roll pad correctly. Check skin condition in each shift and change wet clothes and sheets in time. Use decompression bed, keep the bed unit dry, in the course of hospitalization, patients without pressure sore and other complications. Strengthen turning 
over and massage the limbs of the patients, prevent the formation of deep venous thrombosis, give antithrombotic pressure socks, raise the lower limbs, use an ankle joint pad.

\subsubsection{Nutritional Support}

Patients in a high fever for a long time, lumbar cistern drainage tube continuous external drainage, the body is in a high metabolic state. Intestinal nutrition is a common way of nutritional support, through the supplement of heat, protein, to ensure the body obtain the required nutrients, correct negative nitrogen balance, high metabolic response, improve malnutrition, improve the body's immunity, alleviate intracranial infection [8]. According to the patient's condition and nutritional risk screening assessment, follow the doctor's advice to keep the stomach tube for enteral nutrition fluid support treatment and intravenous hyper nutrition treatment, Q6h the gastric juice withdrawal every day to understand the patient's gastric emptying, avoid sucking sputum or turning over the back before and after nasal feeding to avoid aspiration.

\subsection{Care of Complications}

\section{Intracranial Infection}

Observe the patient's consciousness and vital signs closely, record the patient's body temperature in time, and use appropriate physical cooling measures correctly. Observe and record the color, character, and quantity of drainage fluid in each class, strengthen the nursing care of drainage tube, must strictly implement the principle of aseptic operation. According to the results of cerebrospinal fluid culture and drug sensitivity, adjust antimicrobial agents, use antimicrobial agents according to doctor's instructions, record the effect of medication, and report to the doctor in time.

\section{Results}

The results showed that the patient's condition developed rapidly and abscess rupture had occurred. By closely observing the changes in the patient's condition, he was actively treated with symptomatic treatment, performed brain abscess puncture and drainage in time, avoided brain hernia, and actively controlled intracranial infection after the operation.

\section{Discussion}

\subsection{Brain Abscess Is in Critical Condition and Develops Rapidly, Endangering the Life of the Patient at Any Time}

Patients with brain abscess ruptured into the cerebral ventricle are in critical condition and often suffer from secondary consciousness disturbance, which eventually leads to a high probability of requiring endotracheal intubation and ventilator-assisted breathing [9]. Tseng JH et al. [10] analyzed and believed that timely diagnosis and treatment could affect the prognosis of brain abscesses. However, due to the lack of typical clinical manifestations of some brain ab- 
scesses, antibiotic treatment could make the symptoms disappear, which could easily affect the diagnosis and treatment of the disease. With the development of imaging diagnosis technology, anesthesia technology, antibiotics, and neurosurgical treatment, most brain abscesses can be effectively cured. However, brain abscess is still a very serious fatal disease, which is not treated in time and still has a high mortality and disability rate [11].

\subsection{Timely Surgical Treatment and Rigorous Nursing Are the Keys to the Treatment of Brain Abscess}

This patient is a rare case. On admission, the symptoms were not obvious and the disease progressed rapidly. The occurrence of suppurative meningitis and progressive brain edema aggravated, leading to the deterioration of the disease and difficult treatment. After the patient was admitted to the hospital, the nursing staff evaluated his condition quickly, formulated a targeted nursing plan, closely observed the condition, and cooperated with the doctor to take active antibiotic treatment. After confirming the brain abscess, the doctor has taken puncturing and drainage of the brain abscess in time and combined with sufficient amount and full course of antibiotics treatment according to the drug sensitivity results. After surgery, nursing staff should closely monitor patients, to strengthen the monitoring of the patient vital signs, pus cavity observation and nursing of the drainage tube, discover the symptoms and take the corresponding nursing measures, including tracheotomy nursing care, pipe, heat resistance (infection) care, skincare, observation of complications, and nutritional support, etc.

Because of this kind of disease, we should summarize the nursing experience of patients with brain abscess in domestic and foreign literature, strengthen the monitoring of patients with severe illness, timely detection of disease changes, take nursing measures, for the treatment and prognosis of patients with brain abscess, has important significance.

\section{Conflicts of Interest}

The authors declare no conflicts of interest regarding the publication of this paper.

\section{References}

[1] Baidi, A.H. and Huang, S.L. (2018) Progress in the Diagnosis and Treatment of Brain Abscess. Chinese Journal of Clinical Neurosurgery, 1, 53-55.

[2] Wang, J. (2019) The Cerebral Abscess Was Complicated with a Cerebral Hernia: A Case Report and Literature Review. Electronic Journal of Clinical Medical Literature, 6, 185,187

[3] Zheng, Z., Su, Y.Y. and Yu, A.H. (2005) A Case Report of Brain Abscess Breaking into Ventricle. Chinese Journal of Neuroimmunology and Neurology, 5, 184.

[4] Xu, Y., Lin, J.K. and Feng, H. (2013) Clinical Analysis of Brain Abscess. Journal of the Third Military Medical University, 25, 2042.

[5] Xie, L.N., Xian, J.S., Pan, J.Y., et al. (2010) Nursing Experience of 50 Patients with 
Brain Abscess. Journal of Local Surgery, 19, 63.

[6] Liu, L.H., Wang, Q.X. and Tian, Y.H. (2015) Nursing Care of 56 Patients with Severe Craniocerebral Injury Racheotomy. Chinese Journal of Modern Nursing, 21, 1432-1433.

[7] Chinese Medical Association Branch of Severe Medicine (2013) Guidelines for the Diagnosis, Prevention, and Treatment of Ventilator-Associated Pneumonia. Chinese Journal of Internal Medicine, 52, 524-543.

[8] Xu, N. (2018) Effect of Comprehensive Nursing Intervention Combined with Enteral Nutrition Support on Postoperative Intracranial Infection in Elderly Patients with Severe Craniocerebral Trauma. Gansu Medical Journal, 37, 755-756.

[9] Hou, M. and You, C. (2003) Diagnosis and Treatment of Brain Abscess Ruptured into the Ventricular System. Division 31-233.

[10] Tseng, J.H. and Jeng, M.Y. (2006) Brain Abscess in 142 Patients: Factors Influencing Outcome and Mortality. Neurosurgical Review, 65, 557-562.

https://doi.org/10.1016/j.surneu.2005.09.029

[11] Nathoo, N., Nadvi, S.S., Narotam, P.K., et al. (2011) Brain Abscess: Management and Outcome Analysis of a Computed Tomography Era Experience with $973 \mathrm{~Pa}$ tients. World Neurosurgery, 75, 716-726.

https://doi.org/10.1016/j.wneu.2010.11.043 